\title{
Hippo kinases MST1 and MST2 control the differentiation of the epididymal initial segment via the MEK-ERK pathway
}

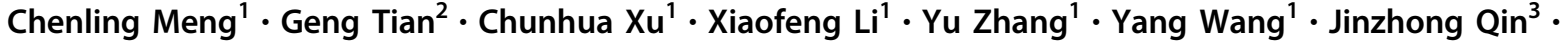 \\ Ellis Kin Lam Fok $\mathbb{D}^{1} \cdot$ Barry T. Hinton ${ }^{4} \cdot$ Kingston King-lun Mak $^{5} \cdot$ Winnie Waichi Shum ${ }^{6} \cdot$ Wai-Yee Chan $\mathbb{D}^{1,7}$. \\ Yin Xia $\mathbb{( i D}^{1,7}$
}

Received: 11 December 2019 / Revised: 3 April 2020 / Accepted: 3 April 2020 / Published online: 24 April 2020

(c) The Author(s), under exclusive licence to ADMC Associazione Differenziamento e Morte Cellulare 2020

\begin{abstract}
Although the roles of the Hippo pathway in organogenesis and tumorigenesis have been well studied in multiple organs, its role in sperm maturation and male fertility has not been investigated. The initial segment (IS) of the epididymis plays a critical role in sperm maturation. IS differentiation is governed by ERK1/2, but the mechanisms of ERK1/2 activation in IS are not fully understood. Here we show that double knockout (dKO) of mammalian sterile 20-like kinases 1 and 2 (Mst1 and Mst2), homologs of Hippo in Drosophila, in the epididymal epithelium led to male infertility in mice. Sperm in the cauda epididymides of mutant mice were immotile with flagellar angulation and severely disorganized structures. Loss of Mst1/2 activated YAP and increased proliferation and cell death in all the segments of epididymis. The mutant mice showed substantially suppressed MEK/ERK signaling in the IS and failed IS differentiation. Deletion of Yap restored the reduced MEK/ERK signaling, and partially rescued the defective IS differentiation and fertility in Mst1/2 dKO mice. Our results demonstrate that YAP inhibits the MEK/ERK pathway in IS epithelial cells, and MST1/2 control IS differentiation and fertility at least partially by repressing YAP. Taken together, the Hippo pathway is essential for sperm maturation and male fertility.
\end{abstract}

Edited by E. Baehrecke

Supplementary information The online version of this article (https:// doi.org/10.1038/s41418-020-0544-x) contains supplementary material, which is available to authorized users.

Yin Xia

Xia.Yin@cuhk.edu.hk

1 School of Biomedical Sciences, Faculty of Medicine, The Chinese University of Hong Kong, Hong Kong, China

2 Bio-X Institutes, School of Medicine, Shanghai Jiao Tong University, Shanghai, China

3 The Key Laboratory of Model Animal for Disease Study, Ministry of Education, Model Animal Research Center, Nanjing University, Nanjing, China

4 Department of Cell Biology, University of Virginia Health System, Charlottesville, VA, USA

5 Guangzhou Regenerative Medicine and Health-Guangdong Laboratory, Guangzhou, China

6 School of Life Science and Technology, ShanghaiTech University, Shanghai 201210, China

7 Key Laboratory for Regenerative Medicine, Ministry of Education, School of Biomedical Sciences, Faculty of Medicine, The Chinese University of Hong Kong, Hong Kong, China

\section{Introduction}

In mammals, spermatozoa enter the epididymis as immotile cells that are incapable of fertilization. The epididymis provides a luminal fluid microenvironment for sperm maturation and storage. Postnatal epididymal development can be divided into three periods, i.e., the undifferentiated period, the differentiation period, and the period of expansion or postdifferentiation. In rodents, the three periods span approximately postnatal day 1 (P1)-P15, P15-P44, and P44-adult, respectively [1]. During the undifferentiated period, the epididymal epithelium consists of undifferentiated low columnar or cuboidal cells with a homologous appearance. During the differentiation period, testicular fluid enters the epididymis [2], and the epithelial cells differentiate into principal, clear/narrow and basal cells, and four epididymal regions, i.e., initial segments (IS), caput, corpus, and cauda are established. The epithelia begin to show region-specific features, for instance, gradients in height with tall columnar epithelial cells and long microvilli seen in IS [3]. During the period of expansion, spermatozoa appear within the epididymal lumen, and epididymal length increases significantly [1]. 
The IS, the most proximal part of epididymis, plays a critical role in sperm maturation. When the IS is not fully developed, sperm show defective maturation with flagellar angulation/hairpin and reduced motility, which may lead to male infertility [4-6]. Therefore, it is important to elucidate the molecular mechanisms underlying IS differentiation.

Two activity levels of the ERK pathway were found in the epididymal epithelial cells: a high level and a basal level. The IS exhibits a highly activated ERK pathway while the basal level is found in the undifferentiated epithelium and remains in regions other than IS from the period of differentiation onward. In response to testicular luminal fluid factors that enter the epididymis during prepubertal development, the ERK1/2 pathway is activated in IS from P17 onward in mice [7, 8], and the activated ERK1/2 pathway governs the IS differentiation and epididymal regionalization [4, 7-9].

In addition to higher activities of the ERK pathway components including RAF1, MEK1/2 and ERK1/2, the IS exhibits higher protein and/or activity levels of Ros1, PTEN, and Src than other epididymal regions $[5,7,10]$. Deficiency in Ros-1 [4, 5, 11], PTEN [6], or Src [10, 12] led to reduced phospho-ERK1/2 levels and prevented prepubertal differentiation of IS, thus placing Ros-1, PTEN, and Src upstream of ERK1/2.

MST1 and MST2 (mammalian sterile 20-like kinases 1 and 2) are the core components of the Hippo pathway. MST1/MST2 activation leads to phosphorylation of YAP and TAZ, the effectors of the Hippo pathway, resulting in their cytoplasmic sequestration and degradation. When Hippo signaling is inactivated, unphosphorylated YAP and TAZ translocate into the nucleus to complex with TEAdomain family members, and thereby activate cell proliferation [13-16].

MST1 and MST2 play important roles in early embryonic development and organ size control. Mice with Mst1 null alleles, Mst2 null alleles, or one copy of either Mst1 or Mst2 were viable and morphologically normal [17, 18]. However, depletion of both Mst1 and Mst2 resulted in embryonic lethality at embryonic day 8.5, suggesting redundant roles of Mst1 and Mst2 [18]. Removal or inhibition of Mst1/Mst2 in newborn or even adult mice resulted in liver enlargement and hepatocellular carcinoma [18-25]. Similarly, in mouse intestines and pancreas, inactivation of Mst1/Mst2 led to intestinal stem cell overproliferation, colonic tumorigenesis and pancreas overgrowth [26-28], suggesting important roles of Mst1/ Mst2 in organ size control and tumorigenesis. Interestingly, our recent study showed that specific deletion of Mst1 and Mst2 in bone inhibited bone formation and bone remodeling [29].

The role of Hippo signaling in the epididymis has not been investigated. In the present study, we found that specific deletion of Mst1/2 in epididymis suppressed the MEK1/2-ERK1/2 signaling pathway and resulted in male sterility due to the failure of IS differentiation and sperm maturation. The phenotypes in double knockout (dKO) mice were partially but significantly rescued by depletion of Yap in the epididymis. Our results identify a new pathway required for the proper IS differentiation and sperm maturation in the mouse epididymis. The results also suggest that the Hippo pathway is essential for sperm maturation and male fertility.

\section{Materials and methods}

\section{Mice}

Mst1 knockout, Mst2 knockout, and Mst1 ${ }^{\mathrm{f} / \mathrm{f}}$; Mst2 ${ }^{\mathrm{f} / \mathrm{f}}$ mouse lines were provided as described $[18,29]$, and $\mathrm{Yap}^{\mathrm{f} / \mathrm{f}}$ mice were purchased from Jackson Laboratory (Stock No.: 027929). To generate Mst1/2 conditional knockout mice, we crossed $\mathrm{Mst}^{\mathrm{f} / \mathrm{f}} ; \mathrm{Mst}^{\mathrm{f} / \mathrm{f}}$ mice with Ksp-cre mice to obtain epididymal epithelial cell Mst1/2 dKO (Mst1 ${ }^{\mathrm{f} / \mathrm{f}}$; $\mathrm{Mst}^{\mathrm{f} / \mathrm{f}} ; \mathrm{Ksp}$-cre) mice. We also crossed $\mathrm{Mst}^{\mathrm{f} / \mathrm{f}} ; \mathrm{Mst}^{\mathrm{f} / \mathrm{f}}$; $\mathrm{Ksp}-\mathrm{Cre}$ mice with $\mathrm{Yap}^{\mathrm{f} / \mathrm{f}}$ to generate Mst1/Mst2/Yap triple knockout (tKO) mice $\left(\mathrm{Mst}^{\mathrm{f} / \mathrm{f}} ; \mathrm{Mst}^{\mathrm{f} / \mathrm{f}} ; \mathrm{Yap}^{\mathrm{f} / \mathrm{f}} ; \mathrm{Ksp}-\mathrm{Cre}\right.$ ). The mice were maintained in a mixed inbred C57BL/6 and $129 / \mathrm{Sv}$ background. All animal studies were approved by The Chinese University of Hong Kong Animal Experimentation Ethics Committee, and were conducted in accordance with The Chinese University of Hong Kong animal care regulations.

\section{Western blotting}

Whole or IS epididymides were homogenized and lysed in TBS $(50 \mathrm{mM}$ Tris- $\mathrm{HCl}, 150 \mathrm{mM} \mathrm{NaCl}$, and $1 \%$ Triton $\mathrm{X}-100, \mathrm{pH}$ 7.4) containing protease and phosphatase inhibitors (Pierce Biotechnology). A total of $25-40 \mu \mathrm{g}$ of protein was separated by SDS-PAGE and transferred to PVDF membranes. The following primary antibodies were purchased from Cell Signaling Technology: phospho-ERK1/2 antibody (\#9101), ERK1/2 antibody (\#9102), phosphoMEK1/2 antibody (\#9154), MEK1/2 antibody (\#9122), MST1 antibody (\#3682), phospho-YAP antibody (\#4911), YAP antibody (\#4912), phospho-RAF1 (S259) antibody (\#9421), phospho-RAF1 (S338) antibody (\#9427), RAF1 antibody (\#53745), phospho-B-RAF antibody (\#2696), PTEN antibody (\#9559), phospho-AKT antibody (S473) (\#4058), AKT antibody (\#9272), phospho-Src (Y527) antibody (\#2105), phospho-Src (Tyr416) antibody (\#6943), Src antibody (\#2109), and phospho-ROS1 (Y2274) antibody (\#3078). MST2 antibody (ab52641) was purchased from Abcam, phospho-c-RAF (Tyr340) 
antibody (PA5-64635) was from Invitrogen, and B-RAF antibody (A2434) was from Abclonal.

\section{Histology and immunohistochemistry (IHC)}

Epididymides and testes were fixed in $4 \%$ paraformaldehyde or Bouin's solution, respectively, overnight at $4{ }^{\circ} \mathrm{C}$, and followed by paraffin embedding and sectioning. Slides were then stained with Harris' hematoxylin and eosin. Tubular thickness was measured by using ImageJ software (imagej.nih.gov/ij/).

For IHC, paraffin-embedded epididymal sections were subjected to antigen retrieval by microwaving in $0.01 \mathrm{M}$ sodium citrate buffer ( $\mathrm{pH}$ 6.0). Sections then were blocked for $1 \mathrm{~h}$ in blocking solution (5\% donkey serum or $3 \%$ goat serum in PBS), and incubated with primary antibodies overnight at $4{ }^{\circ} \mathrm{C}$. The signals were detected using UltraSensitive S-P kit (KIT-9710, MXB Biotechnologies). Sections were counterstained with hematoxylin. YAP antibody (\#4912, Cell Signaling Technology), MST1 antibody (22245-1-AP, Proteintech), MST2 antibody (ab52641, Abcam), and phospho-MEK (\#9154) were used.

\section{$\beta$-galactosidase staining}

Four-week-old epididymides were fixed in 2\% PFA for $1 \mathrm{~h}$ at $4{ }^{\circ} \mathrm{C}$. The tissues were washed with PBS and stained overnight at room temperature in a LacZ staining solution $(0.1 \%$ sodium dexoxycholate, $5 \mathrm{mM}$ potassium ferricyanide, $5 \mathrm{mM}$ potassium ferrocyanide, $2 \mathrm{mM} \mathrm{MgCl}_{2}, 0.01 \%$ $\mathrm{NP}-40$, and $1 \mathrm{mg} / \mathrm{ml} \mathrm{X}$-gal in PBS). The tissues were washed and then fixed in $4 \%$ PFA.

\section{Electron microscopy (EM)}

Caput, corpus, and cauda epididymides were trimmed and fixed in $2.5 \%$ glutaraldehyde in $0.1 \mathrm{M}$ sodium phosphate buffer ( $\mathrm{pH} 7.2$ ) overnight at $4{ }^{\circ} \mathrm{C}$, postfixed in $2 \%$ osmium tetroxide for $1 \mathrm{~h}$, dehydrated in ascending graded ethanol, and embedded in EPON 812 at $60{ }^{\circ} \mathrm{C}$ for $24 \mathrm{~h}$. Ultrathin $60 \mathrm{~nm}$ thick sections were stained with uranyl acetate and lead citrate and examined in a Hitachi $\mathrm{H}-7700$ transmission electron microscope.

\section{Immunofluorescence}

Frozen epididymal sections were treated with $1 \%$ SDS for $4 \mathrm{~min}$ for antigen retrieval. After blocking with 3\% BSA, slides were incubated overnight at $4{ }^{\circ} \mathrm{C}$ with primary antibodies in blocking solution. Sections then were incubated with appropriate secondary antibodies for $2 \mathrm{~h}$ at room temperature, and counterstained with DAPI. PhosphoERK1/2 antibody (\#9101, Cell Signaling Technology),
E-cadherin antibody (\#3195, Cell Signaling Technology), KRT5 (ab52635, Abcam), Ki67 (NB110-89717, Novus Biologicals), AQP9 antibody, or B1-ATPase antibody was used. AQP9 and B1-ATPase antibodies were generously provided by Dr Sylvie Breton.

\section{TUNEL assay}

Apoptotic cells in paraffin sections were stained with ApopTag Plus Fluorescein In Situ Apoptosis Detection Kit (Millipore) following the manufacturer's instructions. The sections were counterstained with DAPI.

\section{Sperm counts, morphology, and motility}

Caudal epididymides were dissected and immersed in $1 \mathrm{ml}$ M2 medium (Sigma-Aldrich). The tissues were minced to allow the sperm to swim into the medium for $10 \mathrm{~min}$ at $37^{\circ} \mathrm{C}$. The samples were diluted with trypan blue staining solution (Bio-Rad), and were transferred onto a hemocytometer to determine the sperm number. For the analysis of sperm morphology, spermatozoa released from cauda epididymides were dispersed on a slide, air-dried at room temperature, and examined in a phase-contrast microscope (Nikon 80i and Ti).

For sperm movie, sperm samples were dispensed in a nonadhesive 24-well plate in sperm washing medium $\left(97.8 \mathrm{mM} \mathrm{NaCl}, 4.69 \mathrm{mM} \mathrm{KCl}, 0.2 \mathrm{mM} \mathrm{MgSO}_{4} .7 \mathrm{H}_{2} \mathrm{O}\right.$, $0.37 \mathrm{mM} \mathrm{KH} \mathrm{K}_{2} \mathrm{PO}_{4}, 2.04 \mathrm{mM} \mathrm{CaCl} 2.2 \mathrm{H}_{2} \mathrm{O}, 4 \mathrm{mM} \mathrm{NaHCO} 3$, $21 \mathrm{mM}$ HEPES, $2.78 \mathrm{mM}$ Glucose, $0.33 \mathrm{mM}$ Na pyruvate, $21.4 \mathrm{mM} \mathrm{Na}$ lactate, and $5 \mathrm{mg} / \mathrm{ml} \mathrm{BSA}, \mathrm{pH} 7.2-7.4$ ) at $37^{\circ} \mathrm{C}$. Movies were recorded on a Nikon Eclipse Ti inverted microscope equipped with a Spot RT Slider Diagnostic Instruments camera, controlled through VisiView software.

\section{Real-time PCR}

Total RNA was isolated from IS of 4-week-old mice or whole epididymides of 8-week-old mice using Mini Best Universal RNA Extraction Kit (TAKARA). First-strand cDNA synthesis was performed using the PrimeScript ${ }^{\circledR}$ RT reagent kit (TAKARA) and amplified using the ABI Prism 7900 Sequence Detection System (PE Biosystems).

\section{RNA sequencing (RNA-seq) and data analysis}

Total RNA was extracted from the ISs of WT, Mst l/Mst2 dKO and Mst1/Mst2/Yap tKO mice at 4 weeks of age using RNA extraction kit (Takara) with DNase I treatment. RNAseq was carried out by Genewiz Inc (Suzhou, China) following standard protocols. The library products were sequenced using an Illumina HiSeq 4000 Genome Analyzer. Standard bioinformatics analysis was performed by 
Genewiz. The RNA-seq data are available in the National Center for Biotechnology Information Gene Expression Omnibus website (http://www.ncbi.nlm.nih.gov/geo) under accession number GSE 138519.

\section{Fertility test}

Seven Mst1 ${ }^{\mathrm{f} / \mathrm{f}} ; \mathrm{Mst}^{\mathrm{f} / \mathrm{f}} ; \mathrm{Ksp}-\mathrm{cre}(\mathrm{dKO})$, five $\mathrm{Mst}^{\mathrm{f} / \mathrm{f}} ; \mathrm{Mst}^{\mathrm{f} / \mathrm{f}}$; $\mathrm{Yap}^{\mathrm{f} / \mathrm{f}}$; Ksp-cre (tKO), or six WT male mice at 6 weeks of age were each mated with a WT C57BL/6J female mouse. The mating pairs were monitored for 6 months (WT and dKO mating) or 10-12 months (tKO mating) to obtain the numbers of pups and litters.

\section{Statistical analysis}

The data presented are means \pm SD of independent replicates $(n \geq 3)$. Student's $t$ test was applied for statistical analysis. All data are represented as means \pm SD of independent replicates $(n \geq 3)$. Comparisons among different segments of the epididymis in Fig. 5b, $d$ were made by oneway ANOVA with Tukey's multiple comparison posttest. $P$ values $\leq 0.05$ were considered statistically significant.

For mRNA analysis by real-time PCR, three ISs of the same genotype were pooled to be one sample. For western blotting, five ISs of the same genotype were pooled to be one sample. The numbers of samples used are described in figure legends. Three animals of each genotype were used for histological and TEM studies. All samples were used in a random manner, and the genotypes are known by the investigators.

\section{Results}

\section{Generation of epididymis-specific Mst1/2 dKO mice}

Single Mst1 knockout male mice and single Mst2 knockout male mice both are fertile, and we did not observe any defects in the structure of epididymis (data not shown), suggesting possible redundant roles of MST1 and MST2 in the epididymis. Therefore, we generated epididymal epithelium-specific Mst1/2 dKO mice by crossing floxed Mst1/2 (Mst1 ${ }^{\mathrm{f} / \mathrm{f}} ; \mathrm{Mst}^{\mathrm{f} / \mathrm{f}}$ ) mice with Ksp-Cre transgenic mice. In the Ksp-Cre transgenic line, Cre is expressed in the epithelial cells of the organs derived from the Wolffian duct including the epididymis, ductus deferens, seminal vesicle, and the kidney collecting ducts [30, 31]. Mst1 and Mst2 mRNA (Supplementary Fig. S1a, b) and protein (both the full length and cleaved forms, Supplementary Fig. S1c-f) levels in the whole epididymis were dramatically reduced in dKO compared with WT mice. Immunohistochemical staining showed that MST1 and MST2 were highly expressed in the epithelial cells of all epididymal segments in WT mice, with the highest expression found in the cauda epididymis for both MST1 (Supplementary Fig. S2a) and MST2 (Supplementary Fig. S2b), and the signals were abolished in Mst1/2 dKO epididymides (Supplementary Fig. S2).

Phosphorylated YAP levels were decreased and total YAP levels were increased in Mst1/2 dKO compared with WT epididymides (Supplementary Fig. S3a, b). As shown by IHC, epithelial YAP signal was much enhanced in all the epididymal segments including the IS in dKO mice as compared with WT mice at ages of 4 (Supplementary Fig. S3c) and 8 weeks (Supplementary Fig. S3d) of age. The YAP target genes Ankrd1 and Cyr61 in the whole epididymis were upregulated in dKO compared with WT mice (Supplementary Fig. S3e).

\section{Male infertility and sperm defects in Mst1/2 dKO mice}

To test fertility, seven dKO and six littermate WT controls at 6 weeks of age were each mated with one WT female for 6 months. All the dKO males did not produce any pups although vaginal plugs were observed in their female partners. The whole epididymis weights were slightly reduced in dKO mice compared with WT mice (Supplementary Fig. S4a), but the overall shape appeared normal. The testis weight (data not shown) and structure (Supplementary Fig. S4b) were normal, indicating spermatogenesis was unaffected.

We then examined the number, motility, and structure of sperm in the cauda epididymis. Sperm counts were slightly reduced in Mst1/2 dKO mice compared with WT mice (Fig. 1a). Sperm from dKO mice showed severe motility defects, with no sperm exhibiting progressive movement (Supplementary movie). Three quarters of the sperm were immotile, and residual motile mutant spermatozoa showed irregular movement. In all, $63.1 \%$ of the mutant sperm displayed flagellar angulation at the annulus, with $44.9 \%$ of hairpin bending and $18.2 \%$ of V-shape (Fig. 1b; Supplementary movie).

As shown by EM, in the midpieces of sperm in WT cauda epididymides, mitochondria were arranged in a cylinder-shaped sheath that wrapped around the outer dense fiber (ODF)-axoneme complex (Fig. 1c-a, c-b). The midpieces in Mst1/2 dKO mice displayed various severe defects including plasma membrane irregularity or rupture, cristolysis or even disappearance of mitochondria, and disappearance of axonemal microtubules (Fig. 1c-d-c-g). Principal pieces showed severe defects including discontinuous and disorganized fibrous sheath, incomplete ODF, and axonemes with missing ODFs and axonemal microtubules (Fig. 1c, compare panels $\mathrm{h}-\mathrm{j}$ with panel $\mathrm{c}$ ). 
A

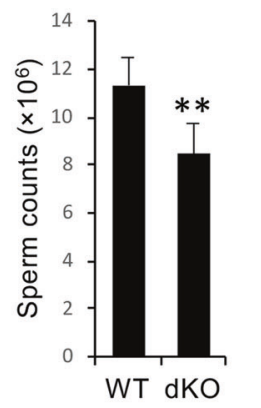

B

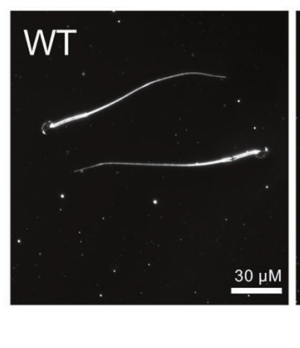

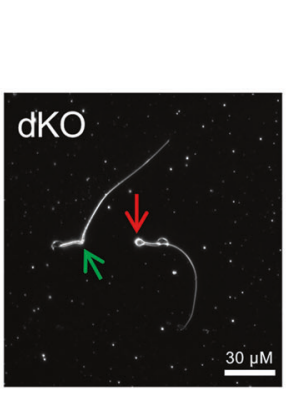

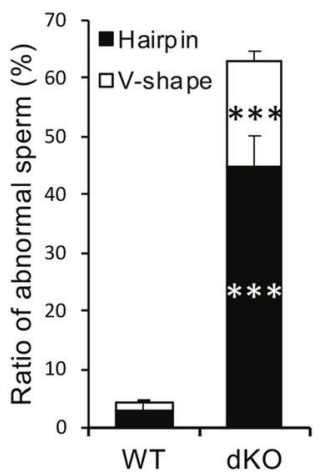

C
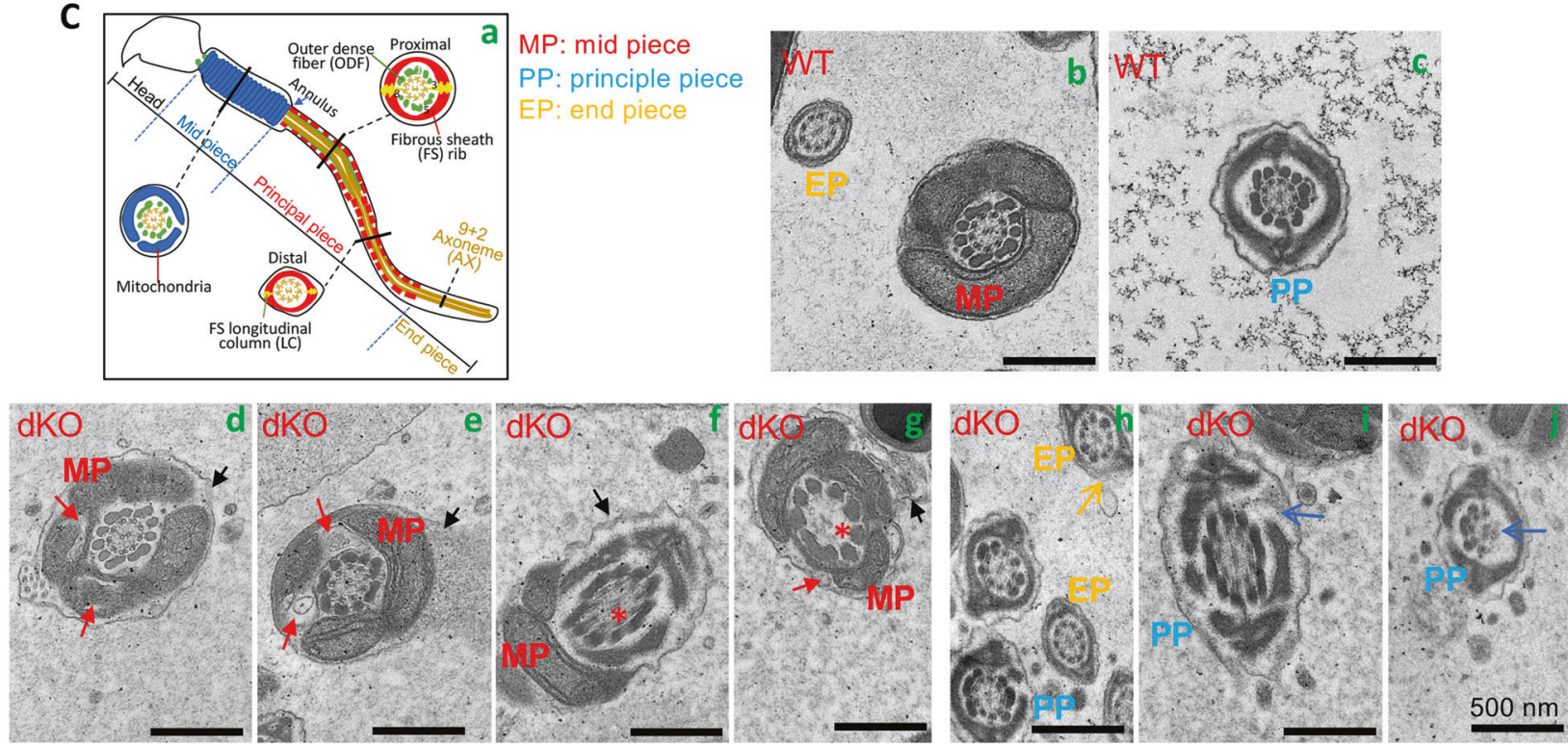

Fig. 1 Sperm maturation defects in Mst1/2 dKO mice. a Number of sperm per caudal epididymis from WT and Mst1/2 cKO mice at 4.5 months of age. The average of the two caudal epididymis of each mouse was used as the number of sperm per caudal epididymis. $n=6 / 4$ (WT/dKO). b Dark field phase-contrast microscopy showing $\mathrm{V}$-shape and hairpin bending of caudal sperm in Mst1/2 dKO mice (left panel). Ratios of sperm with hairpin and V-shape bending were presented (right panel). Three mice were used for each genotype, and 100-200 sperm were analyzed for each mouse. c Electron microscopy (EM) analysis of sperm from the cauda epididymis. c-a Schematic structure of sperm. $\mathbf{c}-\mathrm{b}-\mathrm{j}$ Cauda epididymides from WT and Mst1/2

$\mathrm{dKO}$ mice at 1.5 months of age were used for EM analysis. Cross sections show the ultrastructure of mid-piece (MP), principal piece (PP), and end piece (EP). Note that the MP of dKO spermatozoa shows plasma membrane irregularity or rupture (black arrows), cristolysis (red arrows) or even disappearance of mitochondria, and disappearance of axonemal microtubules (red asterisks). The PP of dKO spermatozoa exhibits discontinuous and disorganized fibrous sheath (FS), incomplete outer dense fiber (ODF), and axonemes with missing ODFs and axonemal microtubules (blue arrows). The EP displays disrupted sperm plasma membrane (orange arrow).

End pieces displayed disrupted sperm plasma membrane (Fig. 1c-h). Thus, loss of Mst1/2 in epididymal epithelial cells results in complex defects in sperm structure and motility, ultimately causing male infertility. These sperm defects indicate drastic alterations in the epididymal epithelial structure and function in Mst1/2 dKO mice. $* * * P<0.001$.

\section{Defective initial segment (IS) differentiation and regionalization in Mst1/2 dKO mice}

Flagellar angulation has been found to be associated with failed prepubertal IS differentiation [5, 6]. We, therefore, analyzed the activity of endogenous $\beta$-galactosidase, a marker for epididymal regionalization, by staining with $\mathrm{X}$-gal. As expected, $\beta$-galactosidase is expressed in a regionalized manner in WT mice at 4 weeks of age, with the strongest staining found in the IS, and faint staining in the caput epididymis. In dKO mice, the strong staining in the IS was absent (Fig. 2a).

At 17-21 days postpartum, the cuboidal IS epithelium differentiates into a tall columnar epithelium. Indeed, WT ISs are characterized by tall columnar epithelial cells, but the height of epithelia was reduced in Mst $1 / 2 \mathrm{dKO}$ mice compared with WT mice at both 4 (Fig. 2b, c) and 8 weeks (Supplementary Fig. S5) of age. Moreover, in WT animals, 


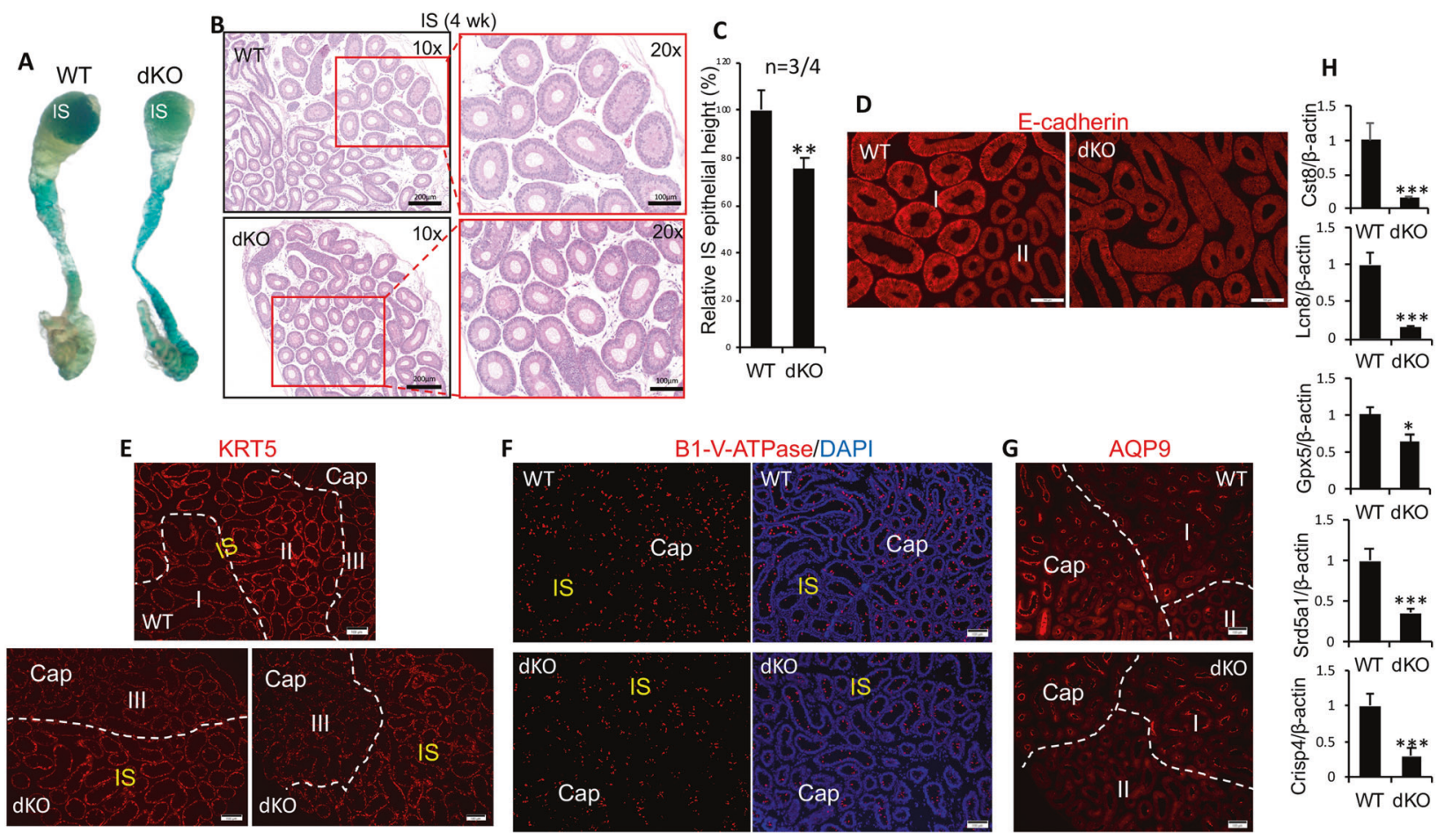

Fig. 2 Loss of initial segment (IS) differentiation and regionalization in Mst1/Mst2 dKO mice. a Epididymides from WT and Mst1/2 $\mathrm{dKO}$ mice at 4 weeks of age were stained with X-gal to visualize endogenous $\beta$-galactosidase activity and epididymal regionalization. $\mathbf{b}$ H\&E-stained paraffin-embedded sections of initial segments (IS) from WT and Mst1/2 dKO mice at 4 weeks of age. c Comparison of epithelial height of region I of initial segment between WT and Mst1/2 $\mathrm{dKO}$ mice at 4 weeks of age. Three WT mice and four dKO mice were used, 4-6 tubules were measured for each epididymis, and five measurements were made for each tubule. $\mathbf{d}-\mathbf{g}$ Immunofluorescent labeling for E-cadherin, KRT5, B1-V-ATPase, and AQP9 in the initial segment

the nucleus was located above a clearly defined basal cytoplasmic zone. In contrast, the nuclei in IS epithelial cells of dKO were often located closer to the basal membrane (Fig. 2b; Supplementary Fig. S5).

To further analyze IS differentiation, we performed immunofluorescence for E-cadherin, KRT5 (a basal cell marker), B1-V-ATPase (a narrow/clear cell marker), and AQP9 (a principal cell marker) on the ISs of mice at 4 weeks of age (Fig. 2d-g). Control IS had a stronger Ecadherin labeling in region I than in region II, while dKO mice showed evenly weak labeling in the corresponding IS region, which was similar in intensity to that in region II of WT mice (Fig. 2d). KRT5 was present in basal cells, which outlined their shape. WT mice showed strong KRT5 labeling in region II and weak labeling in region I of IS. Compared with WT mice, dKO basal cells in the corresponding IS region had intermediate and even KRT5 labeling. No appreciable difference was observed in basal cell shapes between the two genotypes (Fig. 2e). B1-VATPase staining demonstrated similar labeling density and
(IS) and caput (Cap) epididymis. Frozen sections from the epididymides of WT and Mst1/2 dKO mice at 4 weeks of age were used for immunofluorescent staining for E-cadherin (d), KRT5 (e), B1-VATPase (f), and AQP9 (g). DAPI staining was included to show the tubular localization of the B1-V-ATPase signal (f). h mRNA expression of key IS functional genes in the initial segment. Initial segments were collected from WT and Mst1/2 dKO mice at 4 weeks of age. Three initial segments of the same genotype were pooled to be one sample, and used to measure mRNA levels of Cst8, Lcn8, Gpx5, Srd5a1, and Crisp4 by real-time PCR. $n=4$. $\beta$-actin was used as internal control for real-time PCR. $* * P<0.01 ; * * * P<0.001$. positive cell numbers in IS between WT and dKO mice (Fig. 2f). AQP9 staining was present in the apical membrane of region I, but absent in region II of IS in WT mice. Interestingly, as in WT, AQP9 in the IS of dKO also showed differential staining, which allowed us to tell regions I and II (Fig. 2g). mRNAs for Cst8 (CRES), Lcn8 (MEPI7), Gpx5 (GPX), Srd5a1 (steroid $5 \alpha$ reductase), and Crisp4 (epididymal cysteine rich secretory proteins 4) genes, which are expressed in IS and encode proteins important for epididymal functions [32], were profoundly decreased in the IS of Mst1/2 dKO mice compared with the IS of WT mice (Fig. 2h). These data demonstrate that differentiation and regionalization of the IS were severely disrupted in Mst1/2 dKO mice.

\section{Reduced activities of the MEK1/2-ERK $1 / 2$ pathway in the initial segments of Mst1/2 dKO mice}

To understand how deletion of Mst1/2 affected the differentiation of IS and sperm function, we compared the 


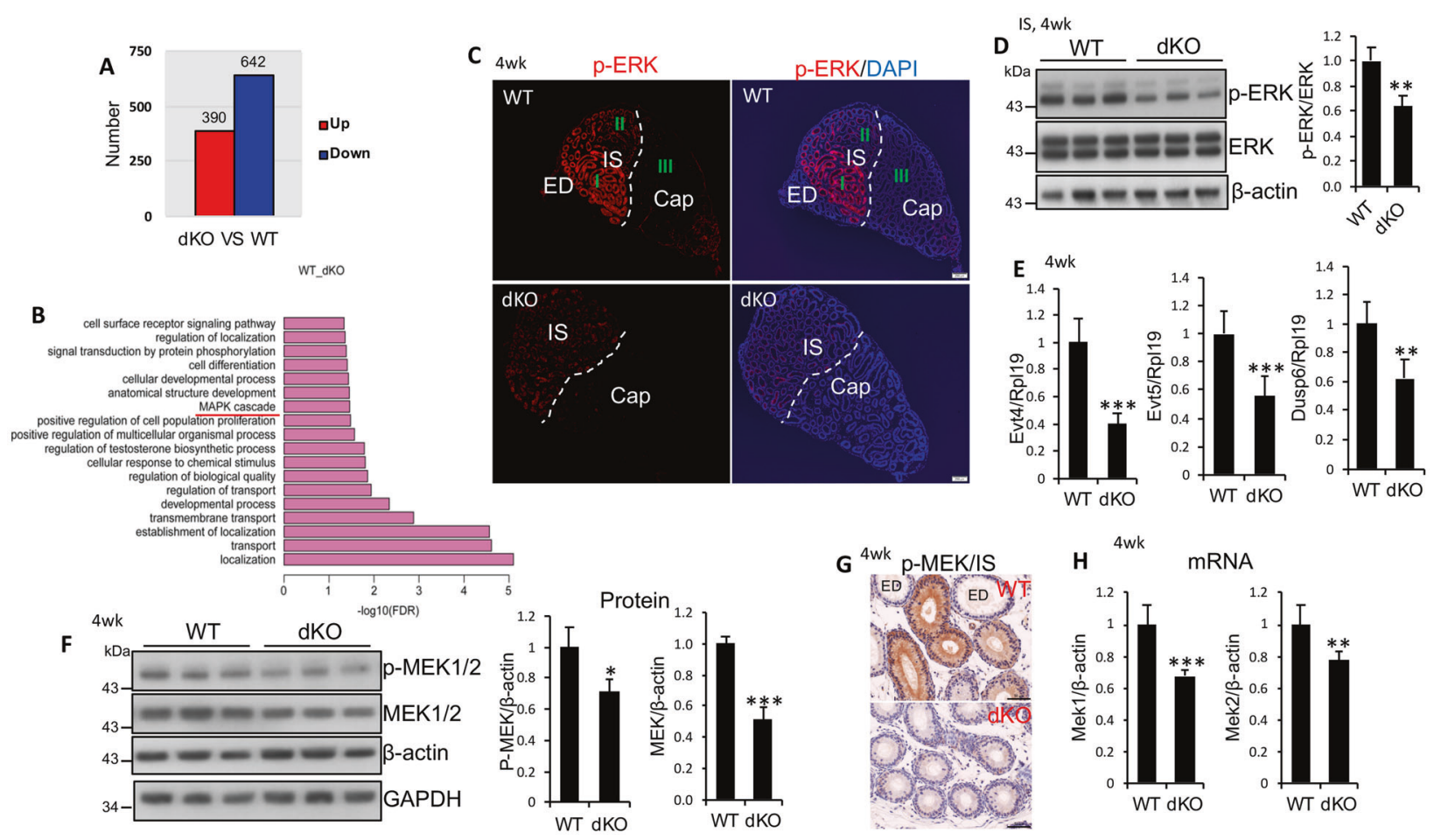

Fig. 3 Inhibition of the MEK/ERK1/2 pathway in the initial segment (IS) by deletion of Mst1/2. a Bar chart of the numbers of differently expressed genes in MST1/2 dKO IS by RNA-Seq. Three hundred and ninety genes (red) were upregulated, and 642 genes (blue) were downregulated. These differentially expressed genes were chosen by $\geq 2$-fold change and $q$ value $<0.05$. b The top GO terms associated with molecular functions of differently expressed genes are shown. $n=3$. c Immunofluorescence for phospho-ERK1/2 (p-ERK1/2) in IS and caput epididymides of WT and Mst $1 / 2 \mathrm{dKO}$ mice at 4 weeks of age. Frozen sections from the epididymides were used for immunofluorescent staining for $\mathrm{p}$-ERK1/2. DAPI staining was also included. d Western blot analysis of p-ERK1/2 in the initial segment. Initial segments were collected from WT and Mst1/2 dKO mice at 4 weeks of age. Five initial segments of the same genotype were pooled to be one sample. Western blotting was performed for p-ERK1/2, ERK1/2, and $\beta$-actin (left panel). Quantitative analysis of $\mathrm{p}$-ERK1/2 relative to ERK1/2 was performed by densitometry (right panel). e mRNA levels

transcriptomic profiles of the ISs from dKO and WT mice at 4 weeks of age using RNA-seq. A total of 1032 genes were identified to be dysregulated, including 642 genes downregulated and the rest (390) upregulated in dKO IS (Fig. 3a). The Gene Ontology (GO) term analysis of all the differently expressed genes (DEGs) in dKO IS revealed a significant enrichment of GO terms related to the MAPK cascade (Fig. 3b). It has been well established that during prepubertal development, the first wave of testicular fluid enters the epididymis and activates the ERK pathway in the IS epithelial cells, and the activated ERK1/2 govern IS differentiation [4, 7-9]. These observations prompted us to study the ERK pathway in the Mst1/2 dKO epididymis. As expected, high phospho-ERK1/2 levels were seen in region I of IS, and the signal was reduced in region II of IS, and even more reduced in region III (proximal caput) in WT mice at age 4 weeks of the ERK1/2 target genes in the initial segment. Initial segments were collected from WT and Mst1/2 dKO mice at 4 weeks of age. Three initial segments of the same genotype were pooled to be one sample and were used to measure mRNA levels of Evt4, Evt5, and Dusp6 by real-time PCR. $n=5$. f Western blot analysis of p-MEK1/2 and MEK1/2 in the initial segment. Initial segments were collected from WT and Mst1/2 dKO mice at 4 weeks of age. Quantitative analysis of $\mathrm{p}$-MEK1/2 relative to $\beta$-actin and MEK1/2 relative to $\beta$-actin was performed by densitometry (right panels). g Immunohistochemistry for $\mathrm{p}-\mathrm{MEK} 1 / 2$ in WT and dKO epididymides. Paraffin epididymal sections from 4-week-old mice were used for immunohistochemistry with DAB staining. Initial segments are presented. h mRNA levels of Mek1 and Mek2 in the initial segment. Initial segments were collected from WT and Mst1/2 dKO mice at 4 weeks of age. $n=5$. ED efferent ducts, IS initial segments, Cap caput epididymis. $* P<0.05 ; * * P<0.01 ; * * * P<0.001$.

(Fig. 3c). In dKOs, phospho-ERK1/2 levels in IS were much reduced compared with the levels in WT although the signal was still stronger than that in region III. No regional difference in phospho-ERK1/2 levels was found within the IS region of dKO epididymis (Fig. 3c). Western blotting on IS lysates confirmed reduced phospho-ERK1/2 levels in dKO compared with WT (Fig. 3d). The downstream genes of the ERK pathway Evt4, Evt5, and Dusp6 in IS were all downregulated in dKO compared with WT mice (Fig. 3e). In contrast, phopsho-ERK1/2 levels in the cauda epididymis did not change between WT and Mst1/2 dKO mice (Supplementary Fig. S6c). These results indicate that the deletion of Mst1/2 reduced the activity of the ERK pathway in IS.

To understand how phospho-ERK1/2 levels were inhibited by Mst1/2 deficiency, we examined the upstream components (Supplementary Fig. S6a). Ros-1 [4, 5], PTEN [6], or Src 


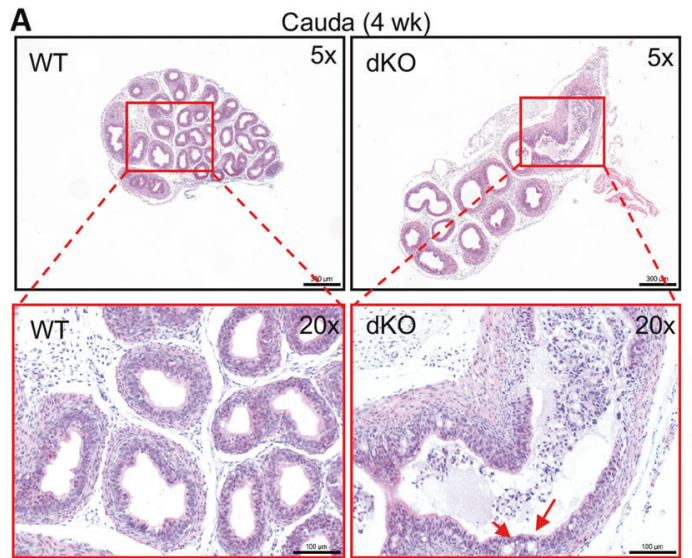

B

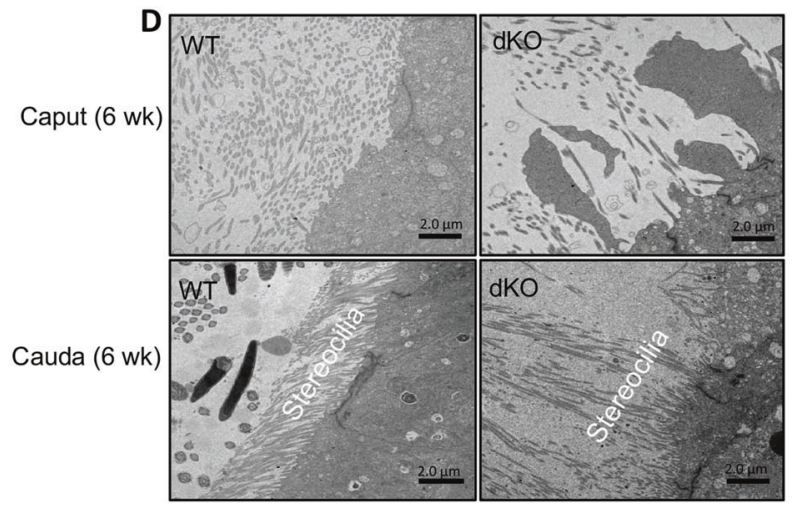

Fig. 4 Morphological changes in the caput and cauda epithelium of Mst1/2 dKO mice. a H\&E-stained paraffin-embedded sections of cauda epididymides from WT and Mst $1 / 2 \mathrm{dKO}$ mice at 4 weeks of age. Note that epithelial cells grew into the lumen and vacuoles (red arrows) were present in the dKO cauda epididymis. b Immunofluorescence for E-cadherin in the cauda epididymis. Paraffin sections from the epididymis of Mst $1 / 2 \mathrm{dKO}$ mice at 4 weeks of age were used for immunofluorescent staining for E-cadherin. White arrows indicate the sloughed cells in the lumen were positive for E-cadherin. c H\&Estained paraffin-embedded sections of cauda epididymides from WT

$[10,12]$ has been found to control IS differentiation through the ERK pathway. However, we did not observe any significant differences in PTEN, phospho-Src (both the stimulatory site Y416 and the inhibitory site Y527), and phosphRos1 levels in IS between WT and Mst1/2 dKO mice. Phospho-c-Raf (both the stimulatory site S338 and inhibitory site S259), phospho-c-Raf (Y340), phospho-b-Raf (S445), and phospho-AKT (S473) levels in IS did not change either between the two genotypes (Supplementary Fig. S6a).

Interestingly, phopho-MEK $1 / 2$ levels in IS were lower in dKO than in WT as shown by western blotting (Fig. 3f) and IHC (Fig. 3g), and this reduction was due to reduced MEK1/2 protein expression (Fig. 3f). Mek1 and Mek2 mRNA levels in IS were also lower in dKO than in WT mice (Fig. 3h). These results suggest that MEK1/2 expression and activity in the IS were inhibited by deletion of Mst1/2.

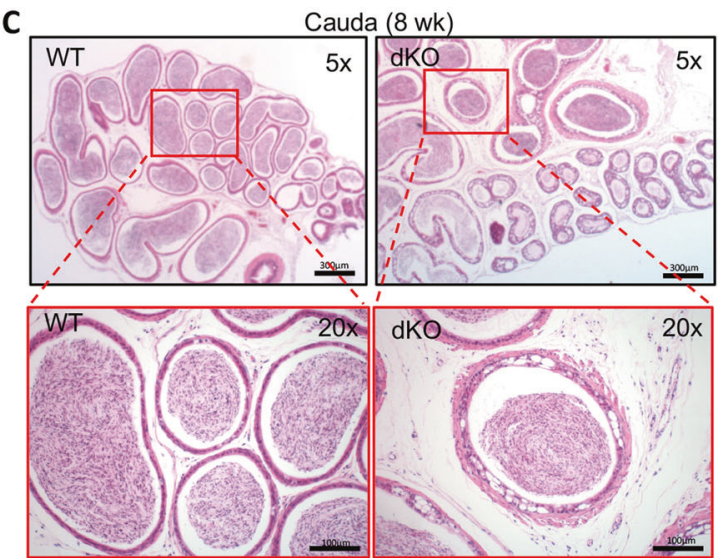

E

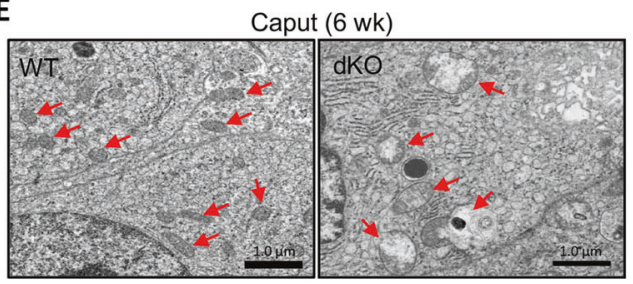

and Mst1/2 dKO mice at 8 weeks of age. Note that there were more vacuoles in the cauda epithelium in dKO mice compared with 4 weeks of age. d Representative electron microscopy photographs of the caput and cauda epididymides of WT and Mst1/2 dKO mice at 6 weeks of age. dKO mice showed giant projections from epithelial cells in the caput and irregular stereocilia in the cauda. e Representative electron microscopy photographs of mitochondrial morphology in caput epithelial cells of WT and Mst1/2 dKO mice at 6 weeks of age. Note that $\mathrm{dKO}$ exhibited mitochondrial swelling and cristolysis.

\section{Progressive alterations in the structures of caput and cauda epididymis in Mst1/2 dKO mice}

The caput epididymides in Mst $1 / 2 \mathrm{dKO}$ mice appeared to be normal in structure at 4 weeks of age (Supplementary Fig. S7a), but displayed epithelial folding and vacuoles at 8 weeks of age (Supplementary Fig. S7b). Mutant cauda epididymides at 4 weeks of age showed more cellularity (Fig. 4a), and the epithelial cells overgrew and were sloughed into the lumen (Fig. 4a, b). Vacuoles were found within the epithelium of $\mathrm{dKO}$ cauda epididymides at 4 weeks of age (Fig. 4a), and more vacuoles at 8 weeks (Fig. 4c). Ultrastructural analysis by TEM showed more irregular and giant protrusions from the apical membrane of epithelial cells in dKO caput epididymides (Fig. 4d). Stereocilia of principal cells in WT cauda epididymides had similar lengths with the apical ends well aligned, while the 


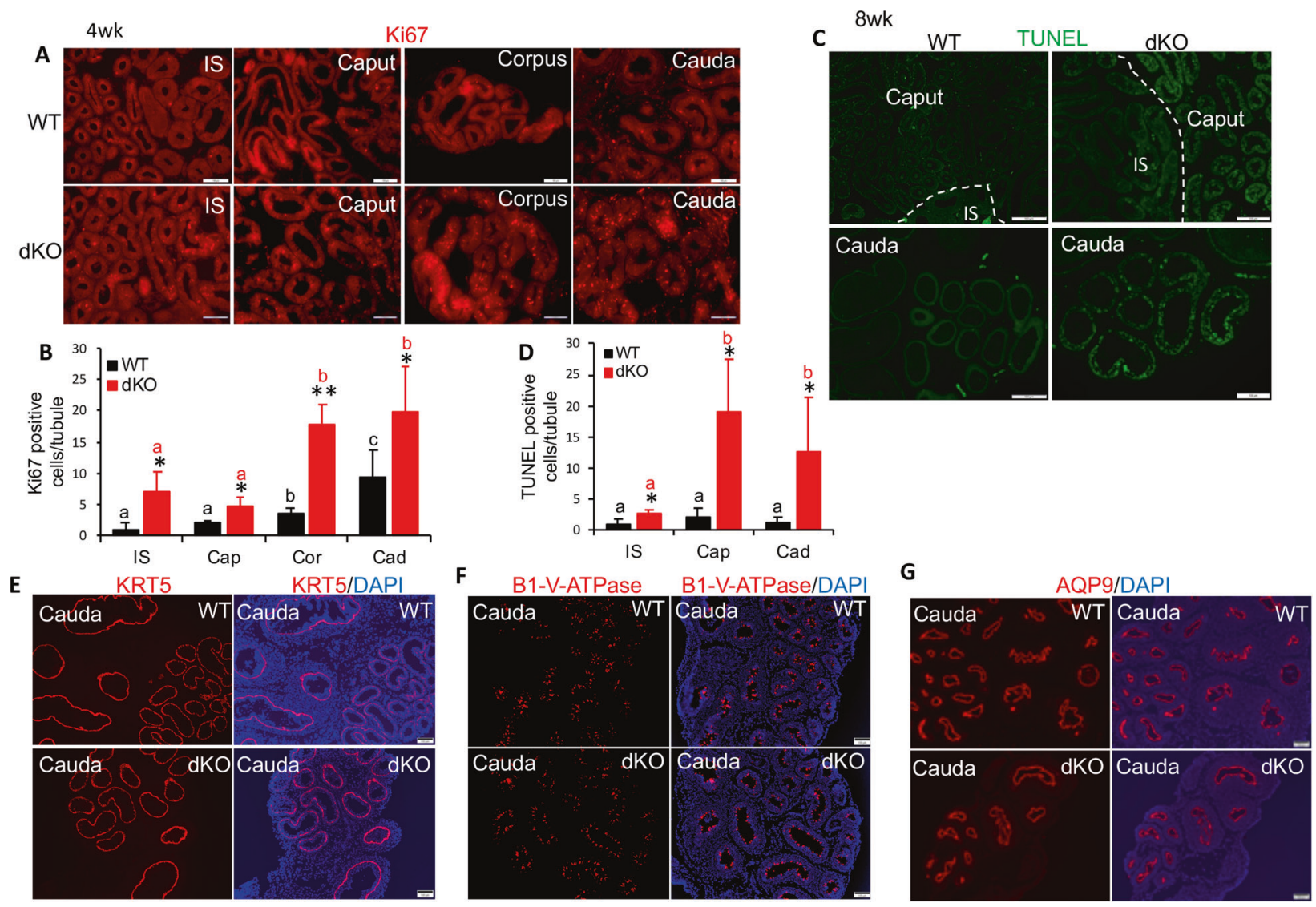

Fig. 5 Changes in cell proliferation, apoptosis and differentiation in Mst1/2 dKO caput and cauda epithelium. a, e-g Epididymides were collected from WT and Mst1/2 dKO mice at 4 weeks of age. Frozen sections were used for immunofluorescent labeling (red) for Ki67 (a), KRT5 (e), B1-V-ATPase (f), and AQP9 (g). DAPI signal was merged with KRT5, B1-V-ATPase or AQP9 signals. b Quantification of positive Ki67 cells in WT and dKO IS, caput, corpus, and cauda epididymides. Three mice were used for each genotype.

stereocilia in $\mathrm{dKO}$ presented both extended and shortened lengths (Fig. 4d). Moreover, the mitochondria in the epididymal epithelial cells of dKO mice exhibited swelling and cristolysis (Fig. 4e).

\section{Increased proliferation and cell death in the epididymides of Mst1/2 dKO mice}

As shown by Ki67 staining, epithelial cell proliferation in IS, caput, corpus, and cauda epididymis was all increased in dKO mice compared with WT mice at 4 weeks of age, with the highest positive cell numbers found in cauda epididymis (Fig. 5a, b). More TUNEL-positive epithelial cells were seen in the caput and cauda epididymides of dKO mice compared with WT mice (Fig. 5c, d). These results may explain the epithelial cell overgrowth and vacuole formation in the Mst1/2 dKO epididymis.

Positive cell numbers and staining patterns for KRT5 (Figs. 2e, 5e), B1-V-ATPase (Figs. 2f, 5f), Foxil (another c TUNEL assay was performed on paraffin sections from the epididymides of WT and dKO mice at 8 weeks of age. d Quantification of TUNEL-positive cells in WT and dKO IS, caput, and cauda epididymides. Three mice were used for each genotype. ${ }^{*} P<0.05$; $* * P<$ 0.01: Comparisons were made between WT and dKO. Letters: means without a common letter are significantly different among different segments of the epididymis in WT (black) or in Mst1/2 dKO (red) mice. Scale bar: $100 \mu \mathrm{m}$.

marker for clear cells, Supplementary Fig. S8), and AQP9 (Figs. $2 \mathrm{~g}, 5 \mathrm{~g}$ ) in caput and cauda epididymides were similar between WT and dKO mice. Therefore, the differentiation of basal, clear, and principal cells in caput and cauda epididymis was not altered by deletion of Mst1/2.

\section{Rescue of the MEK1/2-ERK1/2 pathway, initial segment differentiation, and fertility in Mst1/Mst2/ Yap triple knockout (tKO) mice}

We next generated Mst1/Mst2/Yap tKO mice to determine whether the IS differentiation and fertility phenotypes in Mst1/2 dKO mice were mediated by YAP. As expected, Yap mRNA levels in IS were dramatically reduced by $64.5 \%$ in the tKO mice as compared with the WT or Mst1/2 dKO mice (Fig. 6a). The YAP target genes Ankrd1 and Cyr61 in IS were much lower in tKO mice than in dKO mice, but still higher than in WT mice (Fig. 6a). X-gal staining on the epididymides at 4 weeks of age showed that 
A

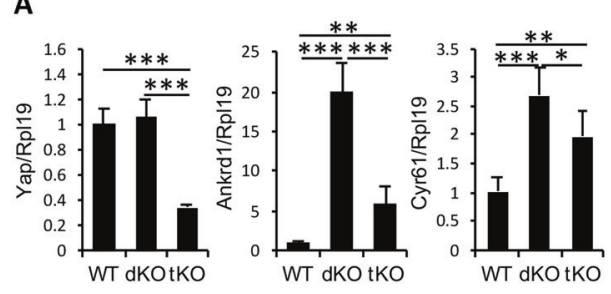

B

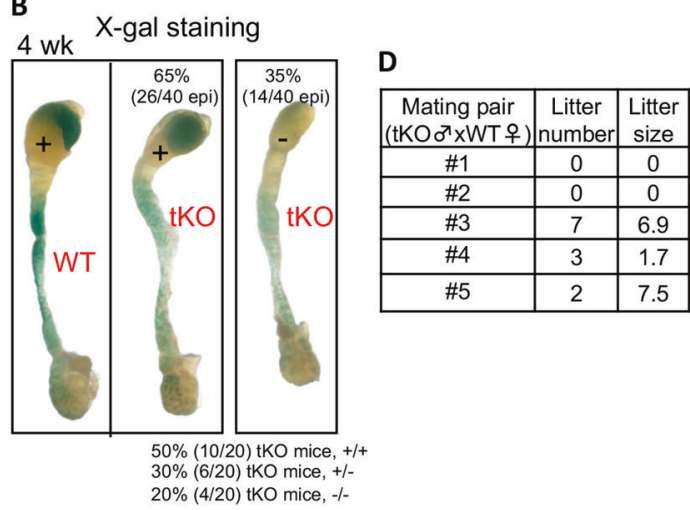

C
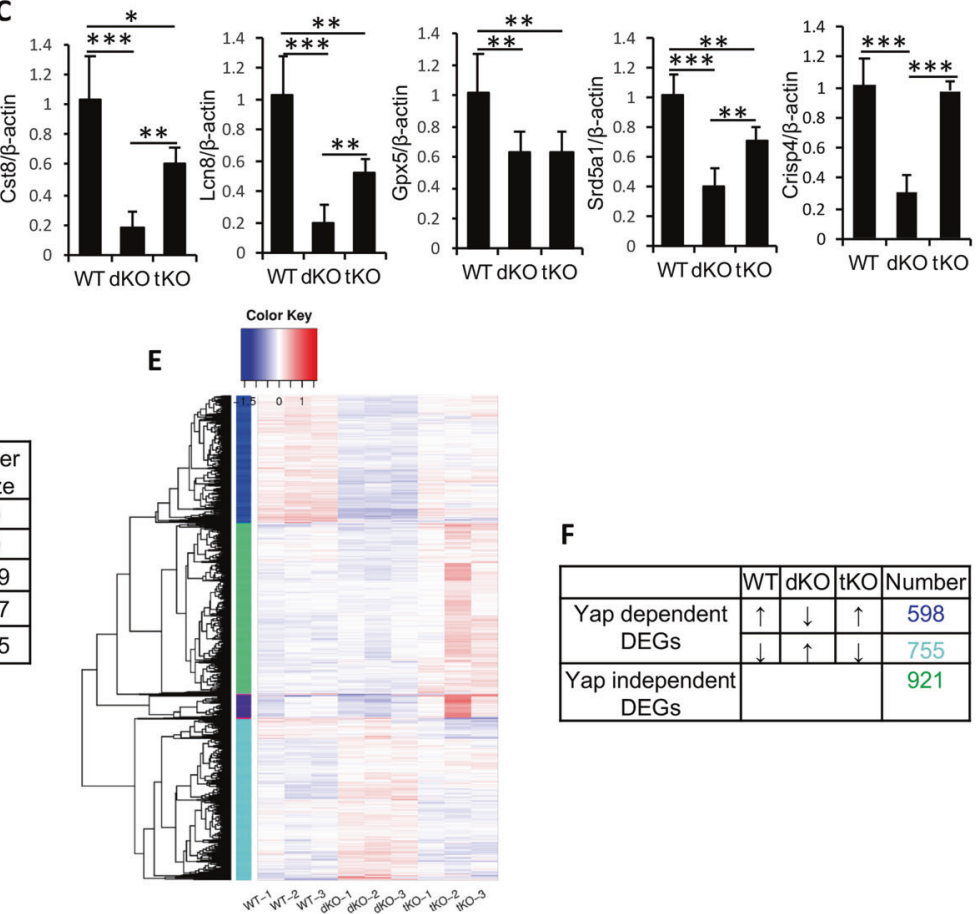

$\mathbf{F}$

\begin{tabular}{|c|c|c|c|c|}
\hline & WT & dKO & tKO & Number \\
\hline $\begin{array}{c}\text { Yap dependent } \\
\text { DEGs }\end{array}$ & $\uparrow$ & $\downarrow$ & $\uparrow$ & 598 \\
\cline { 2 - 5 } & $\downarrow$ & $\uparrow$ & $\downarrow$ & 755 \\
\hline $\begin{array}{c}\text { Yap independent } \\
\text { DEGs }\end{array}$ & & & 921 \\
\hline
\end{tabular}

Fig. 6 Partial rescue of initial segment differentiation and fertility in Mst1/Mst2/Yap tKO mice. a mRNA levels of Yap and the YAP target genes Ankrd1 and Cyr61 in the IS from WT, Mst1/2 dKO and Mst1/Mst2/Yap tKO mice at 4 weeks of age. b Epididymides from WT and tKO mice at 4 weeks of age were stained with $\mathrm{X}$-gal to visualize endogenous $\beta$-galactosidase activity. $\mathbf{c}$ mRNA levels of epididymal functional genes Cst8, Lcn8, Gpx5, Srd5a1, and Crisp4 in the IS of WT, dKO, and tKO mice. d Fertility of the Mst1/Mst2/Yap
tKO mice. Five male tKO mice at 6 weeks of age were each mated with one WT female mouse at the same age, and were monitored for litter numbers and sizes for 10-12 months. e Heat map of 2274 genes that are differentially expressed among WT, dKO, and tKO IS. f The numbers of YAP-dependent and independent genes. $\mathbf{a}, \mathbf{c}$ three initial segments of the same genotype were pooled to be one sample. $n=5 / 5 / 3(\mathrm{WT} / \mathrm{dKO} / \mathrm{tKO}) . * P<0.05 ; * * P<0.01 ; * * * P<0.001$.

may explain why the IS differentiation and fertility phenotypes in Mst1/2 dKO mice were rescued by depletion of Yap.

The MAPK pathway could not be enriched anymore by GO term analysis when WT and tKO profiles were compared (Supplementary Fig. S10). Consistently, immunofluorescent labeling and western blotting showed that the decreased phospho-ERK1/2, phopho-MEK1/2, and MEK1/2 protein levels in dKO IS were largely recovered in tKO mice (Fig. 7a, b). The decreased mRNA levels of Mst1 and Mst2 and the ERK target genes Etv4, Etv5, and Dusp6 in dKO IS were also largely or completely restored (Fig. 7c). Together, these observations indicate that the alterations in MEK1/2-ERK1/2 activities, IS differentiation, and fertility caused by loss of Mst1/2 were largely mediated by Yap.

\section{Discussion}

MST1 and MST2 are the core kinases of the mammalian Hippo tumor suppressor pathway, which are important for control of cell proliferation, apoptosis, and differentiation in various organs such as heart, liver, and intestine [18, 33]. 

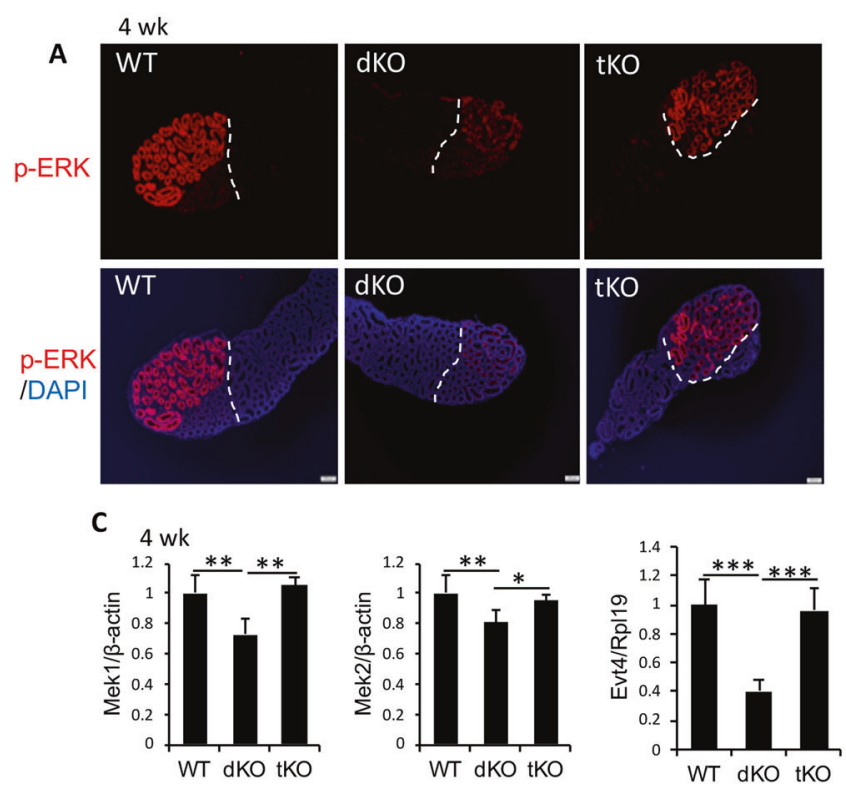

Fig. 7 Recovery of the MEK/ERK pathway in Mst1/Mst2/Yap tKO mice. a Immunofluorescence for p-ERK1/2 in the initial segments of WT, Mst1/2 dKO, and Mst1/Mst2/Yap tKO mice at 4 weeks of age. b Western blot analysis of p-ERK1/2, p-MEK1/2, and MEK1/2 in the IS lysates from WT, Mst1/2 dKO, and Mst1/Mst2/Yap tKO mice (left panels). Quantitative analysis of $\mathrm{p}$-ERK1/2 relative to ERK1/2, p-MEK1/2 relative to GAPDH, and MEK1/2 relative to

However, the role of MST1/2 in male fertility had not been studied. Our data demonstrate that both MST1 and MST2 proteins were highly expressed in epididymal epithelium, and specific deletion of Mst1 and Mst2 in epididymal epithelium resulted in male infertility due to epididymal dysfunction.

During the epididymal differentiation period, epididymal epithelium develops segment-specific gene expression profiles and gains regionalized functions, which are essential for proper sperm maturation and storage. A series of studies demonstrated the importance of epididymal proximal segments (IS and caput) for sperm maturation because defective development or function often leads to male infertility [34]. The epididymal IS exhibits high levels of ERK1/2 activity, which govern IS differentiation [4, 6-9]. Previous studies have demonstrated that Ros-1, PTEN, or Src signals through ERK1/2 to control IS differentiation [4-6, 10, 12]. We now show that the deletion of Mst1/2 in epididymal epithelium blocked the ERK1/2 activation and prevented IS differentiation. Therefore, we identified a new pathway that controls ERK1/2 and IS differentiation.

To determine how Mst1/2 deletion or YAP activation inhibits ERK1/2 phosphorylation, we first screened an array of kinases including b-Raf, c-Raf, PTEN, AKT, Src, and Ros1, which are known to regulate the ERK1/2 pathway, but we failed to see any differences in these kinases between WT and Mst1/2 dKO mice. A previous study
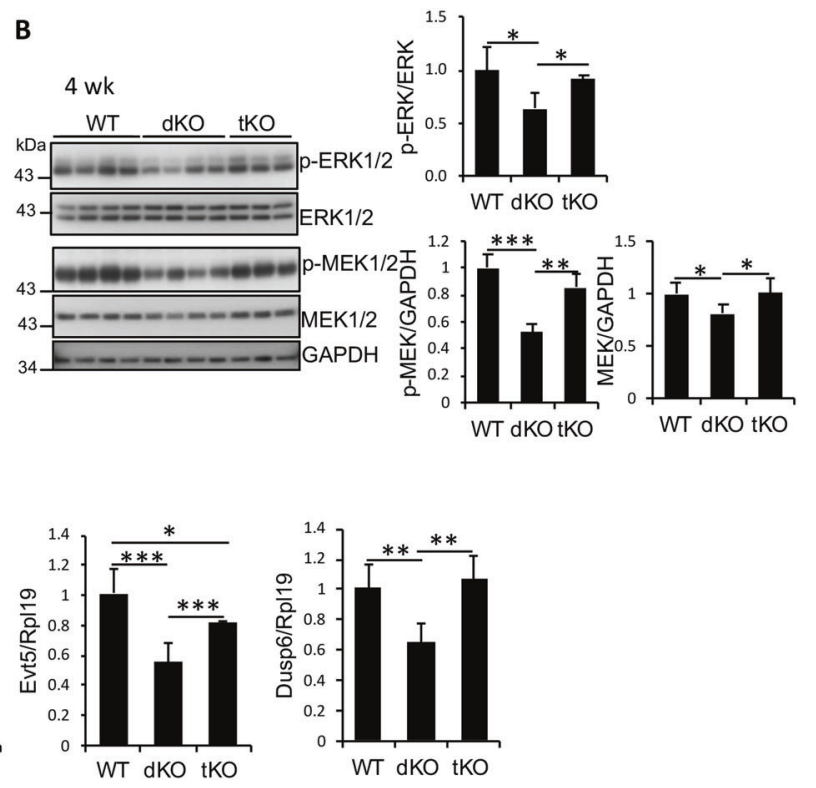

GAPDH was performed by densitometry (right panels). Five initial segments of the same genotype were pooled to be one sample. c mRNA levels of Mek1 and Mek2 and the ERK1/2 target genes Etv4, Etv5, and Dusp6 in the IS of WT, dKO, and tKO. Three initial segments of the same genotype were pooled to be one sample. $n=5 / 5 / 3$ (WT/dKO/tKO). $* P<0.05 ; * * P<0.01 ; * * * P<0.001$.

showed that YAP downregulated PTEN by inducing miR29 to inhibit PTEN translation in MCF10A human breast epithelial cells and in liver tissues [35], but this regulation on PTEN was not found in the ISs of Mst1/2 dKO mice. c-Raf can be activated by Ras and Src. Src phosphorylates c-Raf at tyrosines 340 and 341 [36]. Interestingly, YAP interacted with Src [37]. These observations prompted us to hypothesize that increased YAP in Mst1/2 dKO epididymis may sequester Src, and reduce the availability of Src for c-Raf phosphorylation, thus reducing phospho-c-Raf at Tyr340. MST2 interacted with RASSF proteins and c-Raf (Raf1) [38], thus deletion of Mst1/2 may alter c-Raf phosphorylation. However, we did not see any changes in c-Raf phosphorylation levels at multiple sites in the IS between WT and Mst1/2 dKO. MST1/2 also interacted with the small G protein Rap1 [39], which can activate the b-RafERK pathway [40]. Again, no differences in b-Raf phosphorylation levels in the IS were detected between the two genotypes.

We then moved on to examine the activity of MEK1/2, the kinases immediately upstream of ERK1/2. We found that MEK1/2 mRNA and protein levels in the IS were reduced in Mst1/2 dKO mice compared with WT mice, and as a result, phospho-MEK1/2 levels were also reduced. Interestingly, the decreased MEK1/2, phospho-MEK1/2, and phospho-ERK levels in the IS of Mst1/2 dKO were restored toward the physiological levels by deletion of Yap. 
Therefore, Mst1/2 deletion increased YAP, which, in turn, suppressed the MEK1/2-ERK/12 pathway in the IS.

We attempted to determine the molecular mechanisms by which YAP regulates the MAPK pathway using several cell lines including PC-1 epididymal epithelial cells cultured in a variety of conditions, but we just could not show any regulation of phospo-ERK1/2 by YAP overexpression or MST1/2 knockdown in vitro. As a matter of fact, the regulation of MEK/ERK by YAP took place in the IS but not in the caudal epididymis, indicating this regulation occurs only in certain cellular contexts. This may explain the difficulty in recapitulating the regulation of ERK by YAP in vitro.

Expression of several ERK target genes in the IS was downregulated in Mst1/2 knockout mice, and this downregulation was largely rescued by Yap deletion. These results suggest that YAP may regulate these genes through ERK1/2, but it does no exclude the possibility that YAP may also inhibit these genes independently of ERK1/2.

Despite the restored ERK1/2 phosphorylation levels in Mst1/2/Yap tKO mice, IS differentiation was found only in $65 \%$ of the epididymides and the remaining $35 \%$ were still undifferentiated by the age of 4 weeks. Consistently, the fertility was also only partially rescued by deletion of Yap. Our RNA-Seq analysis revealed that 921 out of 2274 DEGs in the IS of Mst1/2 dKO mice were Yap independent. Even among the 1353 Yap-dependent genes, many were not fully restored in the tKO mice compared WT mice. Therefore, it is possible that the functions of phospho-ERK1/2 in the tKO mice may have been disrupted to various degrees by those genes which are still differentially expressed.

In addition to the defective IS differentiation, a number of other abnormalities were also observed in the initial and other segments. At the microscopic levels, we observed increased cell proliferation and cell death in all the segments, and epithelial cell overgrowth and sloughing in the cauda epididymis of Mst $1 / 2 \mathrm{dKO}$ mice. At the ultrastructural levels, we found irregular stereocilia from the luminal surface of epithelial cells, and mitochondrial swelling and cristolysis in Mst1/2 dKO mice. It is unknown how much these defects contribute to the sperm immotility and infertility in Mst1/2 dKO males. Mice with Ros-1 knockout $[4,5,11]$ or conditional knockout of Pten [6] in the IS each led to failed IS differentiation, which, in turn, resulted in flagellar angulation and infertility. These observations highlight the importance of IS differentiation. However, other defects in the epididymis of Mst1/2 dKO mice may also play a role in the male infertility.

Our results demonstrated that YAP was activated in all the segments of epididymis by depletion of Mst1/2. YAP is known to activate the expression of transcriptional targets involved in cell growth and cell proliferation in multiple cell types. Therefore, it was expected to see higher proliferation activities in the epididymis of the Mst1/2 $\mathrm{dKO}$ mice.
Previous studies have shown that ERK signaling (including the basal and high levels) induces cell proliferation in the epididymis $[4,6,7]$. However, our present study showed that cell proliferation was increased, while the high ERK levels were suppressed in the IS of Mst1/2 dKO mice. More proliferating cells were also observed in dKO cauda epididymis where the basal levels of ERK signaling were not altered. The results argue against that the ERK pathway mediates the increased cell proliferation in the epididymides of Mst $1 / 2 \mathrm{dKO}$ mice.

In summary, we show that the Hippo pathway is essential for maintaining normal IS differentiation and male fertility via modification of the MEK-ERK pathway.

Acknowledgements We thank Dr Sylvie Breton (Massachusetts General Hospital) for providing the AQP9 and B1-ATPase antibodies, Dr Yu Huang (The Chinese University of Hong Kong) for the financial support for RNA-Seq, Dr Wen-Liang Zhou (Sun Yat-Sen University) for PC-1 cells, Dr Xiangjian Zheng (Tianjin Medical University) for the help with X-gal staining, and Sharon Y.C. Ruan (The Hong Kong Polytechnic University) for helpful discussions. We are grateful to Josie Lai and Jean Kung (The Chinese University of Hong Kong) for technical assistance with the electronic microscopy and Corinna $\mathrm{Au}$ (The Chinese University of Hong Kong) for assistance with histology.

\section{Compliance with ethical standards}

Conflict of interest The authors declare that they have no conflict of interest.

Publisher's note Springer Nature remains neutral with regard to jurisdictional claims in published maps and institutional affiliations.

\section{References}

1. Jiang FX, Temple-Smith P, Wreford NG. Postnatal differentiation and development of the rat epididymis: a stereological study. Anat Rec. 1994;238:191-8.

2. Jegou B, Le Gac F, de Kretser DM. Seminiferous tubule fluid and interstitial fluid production. I. Effects of age and hormonal regulation in immature rats. Biol Reprod. 1982;27:590-5.

3. Sun EL, Flickinger CJ. Development of cell types and of regional differences in the postnatal rat epididymis. Am $\mathrm{J}$ Anat. 1979;154:27-55

4. Jun HJ, Roy J, Smith TB, Wood LB, Lane K, Woolfenden S, et al. ROS1 signaling regulates epithelial differentiation in the epididymis. Endocrinology. 2014;155:3661-73.

5. Sonnenberg-Riethmacher E, Walter B, Riethmacher D, Godecke $\mathrm{S}$, Birchmeier $\mathrm{C}$. The c-ros tyrosine kinase receptor controls regionalization and differentiation of epithelial cells in the epididymis. Genes Dev. 1996;10:1184-93.

6. Xu B, Washington AM, Hinton BT. PTEN signaling through RAF1 proto-oncogene serine/threonine kinase (RAF1)/ERK in the epididymis is essential for male fertility. Proc Natl Acad Sci USA. 2014;111:18643-8.

7. Xu B, Yang L, Lye RJ, Hinton BT. p-MAPK1/3 and DUSP6 regulate epididymal cell proliferation and survival in a regionspecific manner in mice. Biol Reprod. 2010;83:807-17.

8. Xu B, Abdel-Fattah R, Yang L, Crenshaw SA, Black MB, Hinton BT. Testicular lumicrine factors regulate ERK, STAT, and NFKB 
pathways in the initial segment of the rat epididymis to prevent apoptosis. Biol Reprod. 2011;84:1282-91.

9. Xu B, Yang L, Hinton BT. The Role of fibroblast growth factor receptor substrate 2 (FRS2) in the regulation of two activity levels of the components of the extracellular signal-regulated kinase (ERK) pathway in the mouse epididymis. Biol Reprod. 2013; 89:48.

10. Xu B, Washington AM, Hinton BT. Initial segment differentiation begins during a critical window and is dependent upon lumicrine factors and SRC proto-oncogene (SRC) in the mouse. Biol Reprod. 2016;95:15.

11. Yeung $\mathrm{CH}$, Sonnenberg-Riethmacher $\mathrm{E}$, Cooper TG. Infertile spermatozoa of c-ros tyrosine kinase receptor knockout mice show flagellar angulation and maturational defects in cell volume regulatory mechanisms. Biol Reprod. 1999;61:1062-9.

12. Krapf D, Ruan YC, Wertheimer EV, Battistone MA, Pawlak JB, Sanjay A, et al. cSrc is necessary for epididymal development and is incorporated into sperm during epididymal transit. Dev Biol. 2012;369:43-53.

13. Pan D. The hippo signaling pathway in development and cancer. Dev Cell. 2010;19:491-505.

14. Yu FX, Zhao B, Guan KL. Hippo pathway in organ size control, tissue homeostasis, and cancer. Cell. 2015;163:811-28.

15. Koo JH, Guan KL. Interplay between YAP/TAZ and Metabolism. Cell Metab. 2018;28:196-206.

16. Misra JR, Irvine KD. The Hippo signaling network and its biological functions. Annu Rev Genet. 2018;52:65-87.

17. Zhou D, Medoff BD, Chen L, Li L, Zhang XF, Praskova M, et al. The Nore1B/Mst1 complex restrains antigen receptor-induced proliferation of naive $\mathrm{T}$ cells. Proc Natl Acad Sci USA. 2008; 105:20321-6.

18. Song H, Mak KK, Topol L, Yun $\mathrm{K}, \mathrm{Hu} \mathrm{J}$, Garrett L, et al. Mammalian Mst1 and Mst2 kinases play essential roles in organ size control and tumor suppression. Proc Natl Acad Sci USA. 2010;107:1431-6.

19. Zhou D, Conrad C, Xia F, Park JS, Payer B, Yin Y, et al. Mst1 and Mst2 maintain hepatocyte quiescence and suppress hepatocellular carcinoma development through inactivation of the Yap1 oncogene. Cancer Cell. 2009;16:425-38.

20. Wu H, Wei L, Fan F, Ji S, Zhang S, Geng J, et al. Integration of Hippo signalling and the unfolded protein response to restrain liver overgrowth and tumorigenesis. Nat Commun. 2015;6:6239.

21. Fan F, He Z, Kong LL, Chen Q, Yuan Q, Zhang S, et al. Pharmacological targeting of kinases MST1 and MST2 augments tissue repair and regeneration. Sci Transl Med. 2016;8:352ra108.

22. Kim W, Khan SK, Gvozdenovic-Jeremic J, Kim Y, Dahlman J, $\mathrm{Kim} \mathrm{H}$, et al. Hippo signaling interactions with Wnt/beta-catenin and Notch signaling repress liver tumorigenesis. J Clin Invest. 2017;127:137-52.

23. Loforese G, Malinka T, Keogh A, Baier F, Simillion C, Montani $\mathrm{M}$, et al. Impaired liver regeneration in aged mice can be rescued by silencing Hippo core kinases MST1 and MST2. EMBO Mol Med. 2017;9:46-60.

24. Hagenbeek TJ, Webster JD, Kljavin NM, Chang MT, Pham T, Lee HJ, et al. The Hippo pathway effector TAZ induces TEAD- dependent liver inflammation and tumors. Sci Signal. 2018;11: eaaj1757.

25. Kim W, Khan SK, Liu Y, Xu R, Park O, He Y, et al. Hepatic Hippo signaling inhibits protumoural microenvironment to suppress hepatocellular carcinoma. Gut. 2018;67:1692-703.

26. Cai J, Zhang N, Zheng Y, de Wilde RF, Maitra A, Pan D. The Hippo signaling pathway restricts the oncogenic potential of an intestinal regeneration program. Genes Dev. 2010;24:2383-8.

27. Zhou D, Zhang Y, Wu H, Barry E, Yin Y, Lawrence E, et al. Mst1 and Mst2 protein kinases restrain intestinal stem cell proliferation and colonic tumorigenesis by inhibition of Yes-associated protein (Yap) overabundance. Proc Natl Acad Sci USA. 2011;108: E1312-20.

28. George NM, Day CE, Boerner BP, Johnson RL, Sarvetnick NE. Hippo signaling regulates pancreas development through inactivation of Yap. Mol Cell Biol. 2012;32:5116-28.

29. Li W, Deng Y, Feng B, Mak KK. Mst1/2 kinases modulate glucose uptake for osteoblast differentiation and bone formation. $\mathrm{J}$ Bone Min Res. 2018;33:1183-95.

30. Shao X, Somlo S, Igarashi P. Epithelial-specific Cre/lox recombination in the developing kidney and genitourinary tract. $\mathrm{J}$ Am Soc Nephrol. 2002;13:1837-46.

31. Schell C, Kretz O, Liang W, Kiefer B, Schneider S, Sellung D, et al. The rapamycin-sensitive complex of mammalian target of rapamycin is essential to maintain male fertility. Am J Pathol. 2016;186:324-36.

32. Cornwall GA. New insights into epididymal biology and function. Hum Reprod Update. 2009;15:213-27.

33. Qin F, Tian J, Zhou D, Chen L. Mst1 and Mst2 kinases: regulations and diseases. Cell Biosci. 2013;3:31.

34. Sipila P, Bjorkgren I. Segment-specific regulation of epididymal gene expression. Reproduction. 2016;152:R91-9.

35. Tumaneng K, Schlegelmilch K, Russell RC, Yimlamai D, Basnet $\mathrm{H}$, Mahadevan $\mathrm{N}$, et al. YAP mediates crosstalk between the Hippo and PI(3)K-TOR pathways by suppressing PTEN via miR29. Nat Cell Biol. 2012;14:1322-9.

36. Marais R, Light Y, Paterson HF, Marshall CJ. Ras recruits Raf-1 to the plasma membrane for activation by tyrosine phosphorylation. EMBO J. 1995;14:3136-45.

37. Pijuan-Galito S, Tamm C, Anneren C. Serum Inter-alpha-inhibitor activates the Yes tyrosine kinase and YAP/TEAD transcriptional complex in mouse embryonic stem cells. J Biol Chem. 2014;289:33492-502.

38. Romano D, Nguyen LK, Matallanas D, Halasz M, Doherty C, Kholodenko BN, et al. Protein interaction switches coordinate Raf-1 and MST2/Hippo signalling. Nat Cell Biol. 2014;16: 673-84.

39. Chang YC, Wu JW, Hsieh YC, Huang TH, Liao ZM, Huang YS, et al. Rap1 negatively regulates the Hippo pathway to polarize directional protrusions in collective cell migration. Cell Rep. 2018;22:2160-75.

40. Uchida M, Enomoto A, Fukuda T, Kurokawa K, Maeda K, Kodama Y, et al. Dok-4 regulates GDNF-dependent neurite outgrowth through downstream activation of Rap1 and mitogenactivated protein kinase. J Cell Sci. 2006;119:3067-77. 